\title{
Polysaccharide-based self-assembling nanohydrogels: an overview on 25- years research on Pullulan
}

\author{
Franco Alhaique*, Pietro Matricardi, Chiara Di Meo, Tommasina Coviello, \\ Elita Montanari
}

\author{
Department of Drug Chemistry and Technologies, "Sapienza” University of Rome, \\ Piazzale Aldo Moro 5, 00185, Rome, Italy.
}

\begin{abstract}
The aim of this overview is to review the evolution of the studies carried out, during more than 25 years, on nanohydrogels obtained by self-assembling of pullulan (PUL) using several hydrophobization strategies. After the first publications, mainly devoted to the preparation and characterization of PUL nanogels, a remarkable number of studies demonstrated how wide can be the field of applications within the main topic of biopharmaceutics. Numerous hydrophilic and lipophilic drugs were entrapped in the nanogel networks, consequently PUL nanogels have been proposed as delivery systems for single drugs and for combination therapies which allowed improvements of pharmacological activities and patient compliance. Furthermore, the large amount of water content allowed loading also proteins which could maintain their native structure and properties. Stimuli-sensitive and stealth PUL nanogel formulations allowed improving the performances of antitumor drugs. These nanohydrogels have also been studied for imaging techniques and for vaccines to be administered by injection and by mucosal application. The studies on PUL nanogels are still in progress and the perspectives for future researches are also addressed.
\end{abstract}

Key words

Pullulan, nanohydrogels, self-assembling, drug delivery, targeting, vaccines 
*Corresponding author at: Department of Drug Chemistry and Technologies, "Sapienza" University of Rome, Piazzale Aldo Moro 5, 00185 , Rome, Italy. Tel.: +39 06 49913605; fax: +39 0649913133. E-mail address: franco.alhaique@uniroma1.it (F. Alhaique)

Other authors:

Pietro Matricardi pietro.matricardi@uniroma1.it

Chiara Di Meo chiara.dimeo@uniroma1.it

Tommasina Coviello tommasina.coviello@uniroma1.it

Elita Montanari elita.montanari@uniroma1.it 


\section{INTRODUCTION}

Natural polysaccharides and their derivatives are indeed among the most employed and versatile polymeric materials that are actually used, or have been proposed, for hydrogel formulations [1]. In this sense it must be pointed out that polysaccharide hydrogels, whether chemically or physically based, show a wide variety of biomedical applications, ranging from their performances as drug carriers to their ability in living cell encapsulation; from bone and cartilage repair to the preparation of friendly scaffolds for tissue engineering. Furthermore, remaining within quite close areas of interest, polysaccharides are often present also in cosmetic and personal care products [2], are used as food additives [3] as well as in agriculture as fertilizer and for herbicide delivery [4], and have even been proposed in formulations for paper and painting cleaning $[5,6]$.

The peculiar and diversified properties of polysaccharides can be related to the different primary structures and molecular weights that these macromolecules may have, as well as to the various type and number of reactive groups that can be present on each single repeating unit. These properties allow numerous chemical modifications that can be appropriately tailored according to the specific use that is assigned to these macromolecules.

It is well known that several natural polysaccharides are capable of forming gels in appropriate conditions. Typical examples are alginate, pectins and gellan, which are able to gel by means of the interactions between the carboxylic acid moieties and divalent ions (i.e., physical gelation by ion complexation); Locust Bean Gum and Xanthan whose synergistic interaction leads to gel formation $[7,8]$ and the stable hydrogels that can be formed by borax interaction with the hydroxyl groups of some polysaccharides [9].

Furthermore, a large variety of bifunctional or multifunctional reagents have been used as crosslinking agents to chemically crosslink hydroxyl and/or other functional groups present on the native polysaccharide chains $[10,11]$.

On the other side it must be pointed out that numerous native polysaccharides are not capable to form hydrogels; moreover, in many cases the performances of hydrogels obtained from natural polysaccharides need significant improvements for their practical applications. For this purpose appropriate chemical modifications can be carried out on the macromolecules. Furthermore, sometimes scientists must resort to multicomponent networks such as semi-Interpenetrating Polymeric Networks (semi-IPNs) and Interpenetrating Polymeric Networks (IPNs) [12].

These networks usually show physico-chemical properties that can remarkably differ from those of the polymeric constituents and their properties, according to specific needs, can be tailored by the choice of the nature and the concentration of the interpenetrating macromolecules [13]. 
Together with the methods used for polysaccharide hydrogel formation, schematically reported above, a relevant number of studies has been focused also to the size of the hydrogel networks for specific applications. Beside the bulk hydrogels, already studied since a long time, during last decades an ever-increasing attention has been devoted towards nanosized hydrogels (nanohydrogels/nanogels). Nanogels can be generally obtained following different techniques such as: polymerization of monomers in homogeneous or nano heterogeneous environments; chemical crosslinking of preformed monomers, top-down methods using lithography, template-assisted nanofabrication and physical self-assembly of interactive macromolecules.

Only few polysaccharides have been exploited for nanohydrogel formation (e.g. hyaluronic acid, chitosan, gellan, scleroglucan, mannans) using different strategies, but in this review the attention will be focused on a single polysaccharide, pullulan (PUL). A wide overview on PUL and its derivatives was recently published with information about the various and diversified reactions that can be carried on this polysaccharide for different applications [14]; however, in the present work, our interest is specifically oriented towards a well defined topic: the self-assembling strategy for PUL nanogel formation and the possible applications of these nanostructures in the field of biomedicine, whose interest and importance is attested by the relevant number of publications that appeared in recent years. Actually, macromolecular self-assembly leading to nanostructures is being investigated since many years [15] because such processes are rather common in nature and also because of the wide variety of practical applications that such systems may have [16], in particular in the field of pharmaceutics.

Different types of hydrophilic and amphiphilic polymer chains have been used for the preparation of nanogels suitable for diagnosis and/or therapy; in the case of polysaccharides, the spontaneous association leading to a nano-hydrogel is often achieved by an appropriate hydrophobic derivatization of these macromolecules [17], according to the general scheme of the process reported in Fig. 1.

According to Kabanov and Vinogradov [16] a nanogel can be defined as "an aqueous dispersion of hydrogel particles formed by physically or chemically cross-linked polymer networks of nanoscale size". Actually, when the self-assembling approach is used, these nano-structures are sometimes defined also as micelles, due to a certain similarity with surfactant micelles bearing a hydrophobic core and a hydrophilic shell [18], and in some studies the value of a critical micelle concentration has been identified and reported, as in the case of one of the first papers on hydrophobized PUL, where the more general term of nano-particles was used.

Throughout this paper the terms nanohydrogel/nanogel and nanoparticle will be used as synonyms while the word micelle will be reported only when the authors of the mentioned articles specifically 
selected this word to indicate the presence of single or multiple microdomains within the nanostructure.

\section{THE LONG STORY OF PULLULAN}

After some papers on the synthesis of cholesterol-modified pullulan (CHP) employed for liposome coating $[19,20]$, and just one year after the wide overview on molecular self-assembly as a strategy for the preparation of nanostructures reported in 1991 in Science [15], J. Sunamoto et al. [21, 22] gave preliminary information on self-aggregation of palmitoyl and cholesterol derivatives of several native polysaccharides such as amylopectin, dextran, mannan and PUL. It was evidenced that the critical concentrations leading to the polymer aggregates were strictly dependent on the degree of substitution of the hydrophobic moiety. Cholesterol-bearing PUL showed a stronger binding for hydrophobic guest molecules and a higher colloidal stability compared with the corresponding palmitoyl-bearing one; for this reason most of the following studies were carried out on CHP. The authors described also the physical cross-linking of cholesterol hydrophobized PUL for the formation of nanoparticles (hydrogels) capable to form a complex with various globular and soluble proteins such as haemoglobin, peroxidase, myoglobin, and cytochrome c.

While in some preliminary approaches CHP was prepared via the aminoethyl-carboxymethylderivative of the polysaccharide followed by the condensation with cholesteryl chloroformate, an improved procedure, which involved the synthesis of cholesteryl $N$-(6-isocyanatohexyl) carbamate and the subsequent condensation with PUL, was proposed in order to increase the stability of the nanogels. In most cases, the self-aggregated nanoparticles have been obtained by sonication in water but they could be prepared also by water dilution of a CHP solution in DMSO. The characterization of the system was carried out by size exclusion chromatography and the critical aggregation concentration was determined by fluorometry. The size and density of the hydrogel nanoparticles were related to the degree of cholesterol substitution. Dynamic Light Scattering (DLS) measurements indicated that the self-assembled structures had an average diameter of $25 \pm 5$ $\mathrm{nm}$, while negative stained electron microscopic observations showed the spherical shape of the particles. In all cases the obtained nanogels were stable in various environments and their size did not change for quite a long time even when kept at room temperature. Furthermore, it has been pointed out that, by appropriate variations of hydrophobic substitution degree and polymer concentration, CHP self-assembling can be controlled from the molecular level (association of hydrophobic groups) to the nanoscale (association of hydrophobized polymers) and macroscopic level (association of nanoparticles), as reported in the scheme of Fig. 2 [23, 24]. 
The last sentence of one of the first papers by Akyoshi et al. [23] stated that "Since it is possible to bring cell specificity to the hydrophobized polysaccharides...they are expected to behave as novel carrier for various lipophilic drugs, proteins and so forth". Actually, the applications of the nanohydrogels obtained following such approach, in particular with PUL, had since then such a wide development that it probably went even beyond the expectations of the authors of that paper. A short time later the same research group reported on the complexation of CHP with $\alpha$ chymotripsin (Chy). The CHP hydrogel strongly complexed with the Chy dimer leading to stable nanoparticles which showed an enhancement of thermal stability in both the structure and the enzymatic activity of the protein, which maintained its activity when released by the complex after addition of Bovine Serum Albumin (BSA). Furthermore, the treatment with pullulanase indicated that the enzyme should be located deeply inside the nanohydrogel network [25].

The mild conditions needed for the spontaneous and easy formation of the complex between the hydrogel nanoparticles and proteins prompted to deeply investigate on insulin loading within the nanohydrogel just by mixing the two components. The supramolecular assembly was very stable, thus the complexed insulin was significantly protected from enzymatic degradation and at the same time aggregation was suppressed. Furthermore, in vivo studies demonstrated that insulin physiological activity was preserved after i.v. injection [26, 27].

Enzymes, such as lipase, were also spontaneously complexed by mixing with the CHP nanogel. The thermal stability of the enzyme was significantly increased and aggregation was prevented even when induced by lyophilization. Size exclusion chromatography suggested that lipase located within the interior of the nanogel, which holds the protein molecule in the segregated nano-matrix by hydrophobic interactions [28] (Fig. 3).

It is well known that in living systems molecular chaperones are capable to selectively trap, by hydrophobic interactions, denaturated proteins or their intermediates, thus preventing irreversible aggregations owing to specific macromolecular host-guest interactions. Similarly, CHP, due to its capacity to entrap proteins, may exert a chaperone-like function, as in the case of the above reported lipase [29]. Such chaperone-like activity for protein refolding using CHP has been studied with several enzymes (e.g. carbonic anhydrase, horseradish peroxidase, citrate synthase). The tested nanogels effectively prevented the protein inactivation by heat, guanidinium chloride or ureainduced denaturation. The enzyme activity was recovered upon dissociation of the gel network in which the proteins were trapped, by addition of cyclodextrins (CD), according to the scheme reported in Fig. 4. Actually, it has been observed that CHP nanogels unfold dramatically in the presence of $\beta$-cyclodextrin: the hydrophobic cavity of $\beta$-CD rapidly screens the cholesterol group 
along the PUL molecules by hydrophobic interactions, and such property suggests also that this hydrogel is suitable for a controlled drug delivery in hydrophobic environments [30 - 33]. The same approach was used to inhibit the formation of amyloid $\beta$-protein fibrils, which are considered as a key step in the pathology of Alzheimer's disease [34].

Due to the fundamental role of CD in the destabilization of the association domains, DLS and Small Angle Neutron Scattering (SANS) were used for a deeper insight on the interaction of CHP with $\mathrm{CD}[35]$ and, more recently, an optical interferometric technique was used to monitor the swelling behavior of CHP gels with different substitution rates when exposed to different types of CD. Obtained results indicated that methyl- $\beta-\mathrm{CD}$ induced the largest swelling changes, while $\alpha-C D$ showed the lowest effect [36].

A recent study, which further extends the horizons of CHP applications, reports on the therapeutic effects that, using calvarian tissues from mouse embryos, can be reached by the use of such nanogel when loaded with the growth factor receptor sFGFR2S252W for the treatment of the Apert syndrome, a congenital disorder characterized by malformations of the skull, face, hands and feet [37].

Although most published papers on PUL-based self-assembled nanoparticles report studies on CHP, also other approaches have been proposed for the same purposes. An example is the PUL hydrophobization by acetylation. PUL acetate spontaneously self-assembled in water forming nanospherical aggregates. The drug-loading study was carried out using clonazepam as a hydrophobic drug and the corresponding release showed a pseudo-zero-order kinetics [38]. A variation of the above reported research was the coupling of the acetate hydrophobized PUL with carboxymethylated-poly(ethylene-glycol). The introduction of PEG was intended to induce stealth properties to the obtained nanoparticles, which were loaded with clonazepam. In vitro delivery experiments indicated that the release mechanism was related to the diffusion of the guest molecule from the core of the nanostructure [39].

Another self-assembled polymeric system based on carboxymethyl-pullulans, varying in the substitution rate on grafted alkyl chains, was proposed for the dissolution of poorly water-soluble drugs. Characterization of the obtained aggregates revealed the presence of hydrophobic nanodomains, which allowed a significant increase of the apparent solubility of benzophenone, while the solubility of docetaxel was only slightly improved because of the surface properties of the drug. In vitro cytotoxicity studies indicated that the new formulation was equipotent to the docetaxel commercial product and, at the same time, that the nano-structure, tested in the absence of the drug, was less cytotoxic against macrophages than the Tween80/ethanol-water system commonly used to increase drug solubility [40]. PUL nanocarriers, intended for nasal and lung 
transmucosal delivery, were proposed by M. Dionísio et al. following a quite different approach. For this purpose, sulphated and aminated PUL derivatives were synthesized and the obtained charged polymers were complexed with chitosan or carrageenan to produce nanocarries, which were loaded with BSA as a model protein [41].

\section{Environmental sensitive PUL nanohydrogels}

In order to induce a thermosensitive response to CHP, the hydrophobized PUL was hydrophobically combined with modified Poly(N-isopropylacrylamide) (HM-PNIPAM). The mixing of CHP and PNIPAM-C ${ }_{18}$ Py yielded, via association of their hydrophobic groups, monodispersed nanogels which above $32{ }^{\circ} \mathrm{C}$ (i.e., the lower critical solution temperature of PNIPAM- $\mathrm{C}_{18} \mathrm{Py}$ ) increased their diameter (from 47 to $160 \mathrm{~nm}$ ), as demonstrated by fluorescence and DLS (Fig. 5 A). This phenomenon was thermoreversible and no aggregation was observed [42, 43].

A reversible and reproducible temperature-induced variation of hydrodynamic radius was observed also with hybrid nanogels obtained from CHP and PNIPAM synthesized by graft free-radical copolymerization of N-isopropylacrylamide (NIPAM) onto methacryloyl-substituted CHP nanogels in the presence of 2,2'-azobis[2-(2-imidazolin-2-yl)propane] as initiator. Depending on the ratio between the two components, different assemblies were obtained. In particular, it is interesting to point out that nanogels with a sufficiently high NIPAM content showed a two-step response to temperature variations (i.e. 35-30 and 35-50) as reported in Fig. 5 B. Such multi-step process was related to the specific grape-like morphology (botryoidal) acquired by the nanogel [44]. In a following study thermosensitive PNIPAM hydrogels were prepared by crosslinking with selfassembled nanogels consisting of cholesteryl- and methacryloyl-substituted PUL. The nanogelcrosslinked PNIPAM showed a rapid deswelling and reswelling behaviour with temperature variations between 20 and $40{ }^{\circ} \mathrm{C}$, as indicated in Fig. 5 C. Such property, which can be controlled, is to be considered useful for releasing hydrophobic molecules and proteins [45].

Within the ever-increasing family of stimuli-responsive delivery systems, together with the above reported thermosensitivity, a self-assembled cholesteryl PUL nanogel sensitive to $\mathrm{pH}$ was also studied. In this case an acid-labile cholesterol (CHOL) derivative was bound to PUL by vinyl ether groups; the obtained product was then hydrolyzed in acidic medium, as reported in Fig. 6 [46]. When complexed with a model protein (BSA) in a neutral environment, the cargo was released at a rate which was controlled by the degradation of the CHOL-PUL grafting moiety. 


\section{Antitumor self-assembled PUL nanohydrogels}

A different approach was followed by $\mathrm{Na}$ et al. [47, 48]: self-organized thermo-responsive nanogels from pullulan-g-poly(L-lactide) were prepared by one-pot method. The nanoparticles, consisting of hydrophobic cores of poly(L-lactide) and hydrophilic outer-shells of PUL, were characterized and the critical association concentration was evaluated. According to the ratio between PUL and poly (L-lactide), products with different properties were obtained and one of them was selected as that with the best performances. Doxorubicin (DOX) was loaded in the nanogels, which showed good encapsulation efficiency. A significant difference in DOX release was observed between 37 and $42{ }^{\circ} \mathrm{C}$ as well as the $50 \%$ inhibitory concentration values evaluated at the two temperatures. According to the obtained results the authors suggested that such nanogels, by means of a triggering temperature, can be used as long-term delivery systems for cancer therapy.

DOX was also loaded in $\mathrm{pH}$-sensitive nanoparticles obtained by conjugation of both urocanic acid (as $\mathrm{pH}$-sensitive moiety) and cholesterol succinate (as hydrophobic moiety). A high drug loading was obtained and results of MTT assays, flow cytometry and confocal microscopy confirmed that such nanostructures were able to realize an intracellular delivery after internalization and a corresponding enhanced cytotoxicity of the drug against MCF-7 cells [49].

A different class of PUL-based self-assembled $\mathrm{pH}$ responsive nanohydrogel was proposed for adriamycin delivery. In this case the nanoparticles were obtained by conjugating sulfadimethoxine to succinylated PUL acetate and the release of the loaded anticancer drug was tested at different $\mathrm{pH}$ conditions. This approach appeared to be suitable for an improved passive accumulation and enhanced drug release at tumor $\mathrm{pH}$ values [50]. Furthermore, such accumulation capability exerted by PUL acetate allowed preparing nanoparticles as effective intratumoral radioisotope $\left({ }^{99 \mathrm{~m}}\right.$ Technetium) carriers for cancer treatment via radiotherapy [51].

Acetate-hydrophobized PUL was used also for paclitaxel incorporation and the obtained selfassembled particles containing the drug were tested both in vitro and in vivo. While in vitro a lower antitumor activity with respect to the free drug was observed because of the sustained release properties of the system, in vivo tests using human colon carcinoma cells, showed a higher reduction in tumor volume [52].

Together with CHP, also $\alpha$-tocopherol PUL polymers (PUTC) showed self-assembling properties. The nanomicelles (as named by the authors), obtained from PUTC by sonication and dialysis, were capable to load 10-Hydroxycamphothecin (HCTP) at different percentages according to the substitution degree (Fig. 7). In vitro experiments indicated that blank PUTCs were biocompatible 
with MCF-7 cells and that the HCTP-loaded nanostructures were more toxic than the free drug due to an increased cellular uptake [53].

More recently, by coupling CHOL to PUL via disulfide bond, reducible nanoparticles (rCHP) were obtained. When loaded with DOX, the nanoparticles released the drug in a reduction-sensitive manner and the growth of HepG2 cells was inhibited. Furthermore, a remarkable antitumor effect was detected in nude mice bearing hepatocellular carcinoma tumors, which was related to an enhanced internalization by receptor-mediated endocytosis and to an accelerated drug release in tumor cells [54].

A completely different approach was followed by other authors who hydrophobized PUL derivatives by conjugating the drug itself on the polysaccharide macromolecule. A combination therapy against hepatocellular carcinoma was proposed by Wang et al. [55]: in this case selfassembling was induced by methotrexate which was conjugated with the $\mathrm{pH}$-sensitive $\mathrm{N}$-urocanyl derivative of PUL. The nanoparticles, obtained by the dialysis method, exhibited a high affinity for specific hepatic cells and were also capable to upload the anti-angiogenic combretastatin A4. By intravenous injection in mice, the new formulation showed a prolonged circulation time in the blood, an increased distribution in the liver and in the tumor cells with respect to the original drug. Another $\mathrm{pH}$ sensitive system was prepared from urocanyl PUL and it was demonstrated that the obtained nanoparticles, when tested as adriamycin carriers, were capable to overcome drug resistance of cancer cells by entering into the nucleus of MCF-7/ADR cells [56].

The above-mentioned methotrexate was studied also for a hepatoma-targeted co-delivery of gene and chemotherapy agent. In this case the methotrexate-hydrophobized PUL did not actually form the main self-assembled structure but only the outer coating of an inner nanoparticle core obtained from the condensation of plasmid DNA expressing green fluorescent protein with poly $(\beta-$ amino)ester. This complex structure exhibited a significant synergic effect on hepatoma cells, evidenced by a high gene transfection capability and an enhanced cytotoxicity, together with strong hepatoma-targeting properties, both at cellular and animal level [57].

Also the DOX molecule was proposed for the polysaccharide hydrophobization. The chemical link of the drug to PUL was obtained through the formation of a $\mathrm{pH}$-sensitive hydrazone bond by reacting the amidated PUL with DOX $\cdot \mathrm{HCl}$. The DOX content in the conjugates increased with the increase of DOX in the reaction. Spontaneous self-aggregation in aqueous media of the PUL-DOX conjugates gave nanoparticles of different sizes according to the initial amidated PUL/DOX $\cdot \mathrm{HCl}$ ratio. The conjugated DOX was released in a pH-responsive manner. PUL, which was present at the shell of the nanoparticles, provided an active targeting to hepatic cells through a specific interaction with asialoglycoprotein receptors on the membrane of the tested cells. Due to such properties, these 
new self-aggregated structures have been considered as suitable tools for liver-targeted antitumor chemotherapy [58].

In order to couple anti-cancer and targeting properties within the same PUL nanohydrogel network, folate-PUL conjugates (FA/PUL), with different degree of substitution, were prepared. The obtained FA/PUL polymers were capable to form spontaneously self-organized networks which showed different DOX-loading and releasing properties related to the degree of FA substitution. Cellular uptake and internalization of the FA/PUL nanohydrogels studies demonstrated that the novel nanogel is suitable for active targeting chemotherapy [59]. FA was also proposed for the decoration of maleilated PUL nanoparticles which were conjugated with DOX (FA-MP-DOX). The obtained system was more effective against ovarian carcinoma cells that the free drug [60]. Furthermore, when pyrroditiocarbamate was also loaded in the FA-MP-DOX nanostructures, a significant increase of activity against DOX-resistant ovarian carcinoma cells was observed, thus indicating that such formulation may have important clinical implications to overcome multidrug resistance [61].

The targeting properties of FA/PUL were also exploited for a photodynamic therapy based on the conjugation of a photosensitizer (pheophorbide-a) with FA/PUL. When such nanohydrogels, with self-quenchable photoactivity, were co-incubated with HeLa cells, a noticeable increase in photoactivity was detected; furthermore, after subcutaneous injection in mice a significant phototoxicity, which became negligible in darkness, was observed [62].

Since several studies revealed that biotin receptors are over-expressed on several tumors characterized by rapid and aggressive growth, biotin-modified CHP nanogels have been proposed as anticancer drug carriers. When loaded with mitoxantrone the obtained self-aggregated structures showed, after an initial burst effect, a sustained release of the drug [63].

Another approach for drug targeting, in this case for protein delivery to myeloma cells and primary $\mathrm{T}$ lymphocytes, both with low heparin sulphate expression, was proposed using cationic $\mathrm{CPH}$ nanogels (cCHP) which were previously described for intracellular BSA delivery [64]. Obtained results indicated that the anti-apoptotic protein Bcl- $\mathrm{x}_{\mathrm{L}}$ delivered by cCHP efficiently blocked apoptosis of the tested cells, suggesting that this cationic nanogel can represent a useful tool to deliver proteins for functional regulation of cells that poorly express heparin sulphate [65]. It interesting to point out that, taking advantage from the possibility of obtaining an intracellular delivery, as above reported, it has been possible to use CHP modified with amino groups as novel carriers of quantum dots. The effective internalization of such nanogel-quantum dot hybrid nanoparticles into several human cells demonstrated that cellular uptake was significantly higher with respect to other conventional carriers and that the new system can be useful as a fluorescent 
probe for bioimaging $[66,67]$. Taking into account that human mesenchymal stem cells (hMSCs) have been found to improve cancer therapy and diagnosis due to their tumor-homing properties [68], early tumors $\left(\sim 3 \mathrm{~mm}^{3}\right)$ were detected, using magnetic resonance and optical imaging both in vitro and in vivo, by means of PUL-deoxycholic acid-diethylene triamine pentaacetic gadolinium-chelate nanoparticles photochemically internalized in hMSCs [69].

\section{PUL nanohydrogels for protein/gene/vaccine delivery}

Together with other polymeric nanohydrogels, also PUL based self-assembled networks have been proposed as vaccine delivery systems [70].

Taking into account that the overexpression of human epidermal growth factor receptor 2 (HER2) is often detected in adenocarcinomas such as breast, ovarian, lung, and gastric cancer, humoral immune responses were studied in patients vaccinated with HER2 oncoprotein complexed with CHP (CHP-HER2) and with CHP-HER2 plus granulocyte-macrophage colony-stimulating factor (GM-CSF). The formulations, which were considered safe, successfully induced specific $\mathrm{CD} 8^{+}$and $\mathrm{CD}^{+} \mathrm{T}$ cells responses against HER2 ${ }^{+}$tumors and GM-CSF seemed to accelerate such responses [71 - 73].

CHP was used also in combination with New York-esophagus-1 (NY-ESO-1). Obtained results, carried out in vitro, showed that CHP/ESO-loaded dendritic cells efficiently stimulated CD8 and CD4 cells, thus indicating that such system can represent a promising polyvalent vaccine for immunotherapy targeting NY-ESO-1 [74].

Following such encouraging results a phase I clinical trial was designed to evaluate the safety, immune response and tumor response in esophageal cancer patients, malignant melanoma and prostate cancer patients. The use of whole protein, containing multiple CD4 and CD8 epitopes, was considered beneficial for cancer vaccines to prevent tumors from evading to immune response, and in most of prostate cancer patients, PSA values stabilized during vaccination [75].

In a following study, vaccines of NY-ESO-1 and HER2, both complexed with CHP, were tested. The results obtained with such vaccine combination indicated, in esophageal cancer patients, that the responses to NY-ESO-1 were comparable to the single vaccine, while for HER2 only fewer antibody responses were observed [76]. More recently, clinical trials, carried out on patients with antigen-expressing esophageal cancer, confirmed the safety and immunogenicity of the NY-ESO-1CHP complex and demonstrated that, after subcutaneous vaccination with the higher tested dose $(200 \mu \mathrm{g})$, immune responses were efficiently induced with better survival benefits [77]. 
Taking into account that a variety of cytokines have proved to be effective in inducing immune responses to eliminate tumor cells, CHP was proposed also as a drug delivery system for tumor immunotherapy by IL-12 sustained release. Repetitive subcutaneous administrations of IL-12 loaded CHP induced, in the tested mice, a drastic delay in the growth of fibrosarcoma which was not observed with IL-12 alone [78].

Since mucosal vaccination is considered a suitable and practical route of immunization capable to induce effective immunity to infectious diseases, CHP nanogels were also tested as antigen delivery systems for nasal vaccines.

According to such purpose a complex formed by a cationic cholesteryl group-bearing PUL above described (cCHP) [65] and a non-toxic subunit fragment of Clostridium botulinum type A neurotoxin (BoHc/A) was intranasally administered to mice. The cCHP-BoHc/A system was effectively taken up by mucosal dendritic cells and vigorous BoHc/A neutralizing serum IgG and secretory IgA antibody responses were induced without co-administration of mucosal adjuvants [79].

Following a similar approach, a pneumococcal vaccine was developed which consisted in the complex formed by cCHP with pneumococcal surface protein (PspA). The efficacy of such cCHP/PspA nasal vaccine was tested in murine pneumococcal airway infection models. Intranasal vaccination with this new nanogel-based complex provided protective immunity against lethal challenge with Streptococcus pneumoniae, prevented colonization and invasion by bacteria in the respiratory tract, induced systemic and nasal mucosal Th17 responses. In both reported studies, no signs of BoHc/A or PspA delivery to olfactory bulbs and central nervous system were detected [80]. A review on nanogel-based systems suitable for antigen delivery by mucosal application, published in 2013, underlines the wide possibilities represented by this type of vaccines for the prevention of infectious diseases [81].

A more recent study demonstrated that CHP is also suitable for selective vaccine delivery to medullary macrophages, selective vaccination behaving as a stealth nanoparticulate delivery system. Obtained results, schematically reported in Fig. 8, together with the parallel observation that medullary macrophages have a potential to effectively induce specific $\mathrm{CD} 8^{+} \mathrm{T}$ cell response to vaccine, represent, according to the authors, an important step for the design of macrophageoriented cancer vaccines with high potency [82].

The possibility to stabilize proteins by their loading in nanohydrogel structures was exploited also for the improvement of etanercept effect in rheumatoid arthritis treatment. The approach was based on a temperature-induced modulation of non covalent interactions between a negatively charged amphiphilic PUL derivative (succinylated PUL-g-oligo(L-lactide)) and the positively charged 
etanercept. The obtained nano-system improved the long-term stability of the protein and, when tested in vivo on rats, allowed a prolonged release and an outstanding therapeutic activity in the arthritis animal model after subcutaneous administration [83].

The success of SiRNA for gene therapy and the need of appropriate carriers capable to provide specific cells with therapeutic concentrations of such macromolecules induced to investigate the possible use of PUL derivatives for a liver-targeted SiRNA delivery. For this purpose polyethylenimine-PUL (PPE) was tested and the obtained nanoparticles, when loaded with fluorescein-labeled SiRNA, led to an increased fluorescence level in the liver of mice with respect to other organs, after systemic injection. Such result, together with the low toxicity detected for the carrier, suggests that the system can be considered as a useful tool for an efficient delivery of SiRNA to the liver [84]. Furthermore, it was pointed out that PPE shows excellent blood compatibility and good transfection efficiency of pGFP and pGL3 on HepG2 cells also in the presence of serum [85].

\section{Scaffolds from PUL nanohydrogels}

The possibility to entrap, within PUL nanogels, proteins which maintained their native structure and properties, allowed proposing such structures as tools suitable for tissue regeneration. For this purpose acryloyl-group-bearing CHPs (CHPA) were synthesized by DCC-mediated condensation; then, after bone morphogenetic protein (BMP2) loading into the obtained nanogel, an aggregation process was carried out which led to the formation of a "nanogel in hydrogel" structure.

Implantation of such gel induced osteoblastic activation, new bone formation in vivo and allowed to heal calvarian defects which did not heal spontaneously [86]. Similarly, complete healing of mice calvarian defects were obtained by co-administration of two bone growth factors (FGF18 and BMP2) embedded in a hydrogel matrix prepared by aggregation of CHPA [87]. Following an analogous approach, a multiarmed poly(ethylene glycol) (PEGSH) was used as a crosslinker for the building blocks of CHPA leading to a physically crosslinked network formed by self-assembly of amphiphilic polysaccharide and a chemically crosslinked network among the nanogels (NanoCliP) [88]. The porous NanoCliP was able to trap proteins (e.g. insulin), liposomes and cells; furthermore, when subcutaneously transplanted into mice, it stimulated cell infiltration, tissue ingrowth and neovascularization [89]. Due to all these properties, the NanoCliP gel, according the authors, "represents a new universal platform as a scaffold for tissue engineering" 


\section{CONCLUSIONS}

Although this overview is intentionally limited to a single polysaccharide and to its hydrophobization which leads to nanohydrogel formation by self-assembling, it is evident, from the above reported results obtained during the last twenty-five years, how wide can be the use of such nanoparticles within the field of pharmaceutical applications. Beside the different self-assembling strategies that can be followed, PUL nanogels have been shown as suitable carriers for small biologically active agents as well as for biomacromolecules. Due to the extremely high water content of the hydrogel environment and the mild loading conditions, entrapped proteins can maintain their native overall structure and properties and, in case, exert also chaperone-like functions. At the same time the hydrophobic microdomains present in these structures allow to increase the solubility and efficacy of poorly soluble substances. Consequently, PUL nanogels have been proposed as targeting and delivery systems for single drugs and for combination therapies which can lead to improvements of pharmacological activities and patient compliance. Stimulisensitive PUL nanogel formulations together with the possibility to induce, when needed, stealth properties to the nanoparticles, allowed to enhance the performances of antitumor drugs; furthermore, these nanohydrogels have also been studied for imaging, for vaccines to be administered by injection and by mucosal application and as scaffolds for tissue engineering. Despite the relevant amount of publications on PUL nanogels it can be stated that we are not at the end of the story: further improvements can still be reached with this multitasking nanostructure which should sometimes be combined with parallel studies. As an example, a more accurate identification of immunological biomarkers, together with the improvement of nanogels complexed with antigens, should allow a better evaluation of the clinical response to cancer immunotherapy. Also the combination of imaging with therapeutic applications (theranostics) still appears to be only at its early stage and needs to be further developed and several "last generation" drugs and vaccines, as those obtained through DNA recombinant techniques, could probably be fruitfully administered using this type of nanogels. Moreover, it can be stated that the wide experience acquired with PUL systems should be more systematically applied also to other polysaccharides, thus leading to even better performances of these nanostructures. 


\section{REFERENCES}

[1] T. Coviello, P. Matricardi, C. Marianecci, F. Alhaique, Polysaccharide hydrogels for modified release formulations, J. Contr. Rel. 119 (2007) 5-24.

[2] H. Lautenschläger, (Poly) Saccharides in cosmetic products-From Alginate to Xanthan Gum, Kosmetische Praxis 4 (2009) 12-15.

[3] R. Shepherd, Polysaccharides, in: J. Smith, L. Hong-Shum (Eds.) Food Additives Data Book, Second Edition, Whiley-Blackwell, Oxford, UK, 2011, pp. 683-751 doi: 10.1002/9781444397741.ch10.

[4] E.V.R. Campos, J.L. De Olivera, L.F. Fraceto, B. Sing, Polysaccharides as safer release systems for agrochemicals, Agron. Sustain. Dev. 35 (2014) 47-66.

[5] E. Carretti , M. Bonini , L. Dei, B. H. Berrie, L.V. Angelova, P. Baglioni, R.G. Weiss, New frontiers in materials science for art conservation: responsive gels and beyond, Acc. Chem. Res. 43 (2010) 751-760.

[6] C. Mazzuca, L. Micheli, E. Cervelli, F. Basoli, C. Cencetti, T. Coviello, S. Iannuccelli, S. Sotgiu, A. Palleschi, Cleaning of paper artworks: development of an efficient gel-based material able to remove starch paste, ACS Appl. Mater. Inter. 6 (2014) 16519-16528.

[7] D.F. Zhan, M.J. Ridout, G.J. Brownsey, V.J. Morris, Xanthan-locust bean gum interactions and gelation, Carbohydr. Polym. 21 (1993) 53-58.

[8] C. Sandolo, D. Bulone, M.R. Mangione, S. Margheritelli, C. Di Meo, F. Alhaique, P. Matricardi, Synregistic interaction of Locust Bean Gum and Xanthan investigated by rheology and light scattering, Carbohydr. Polym. 82 (2010) 733-741.

[9] T. Coviello, M. Grassi, R. Lapasin, A. Marino, F. Alhaique, Scleroglucan/borax: characterization of a novel hydrogel system suitable for drug delivery, Biomaterials 24 (2003) 2789-2798. 
[10] K. Park, W.S.W. Shalaby, H. Park (Eds), Biodegradable hydrogels for drug delivery, Technomic Publishing Co. Lancaster PA, 1993 pp. 67-98.

[11] W.E. Hennink, C.F. van Nostrum, Novel crosslinking methods to design hydrogels, Adv. Drug. Deliv. Rev. 64 (2012) 223-236.

[12] A.D. Jenkins, P. Kratochvìl, R.F.T. Stepto, U.W. Suter, Glossary of basic terms in polymer science (IUPAC Recommendations 1996), Pure Appl. Chem. 68 (1996) 2287-2311.

[13] P. Matricardi, C. Di Meo, T. Coviello, F. Alhaique, W. Hennink, Interpenetrating polymer networks polysaccharide hydrogels for drug delivery and tissue engineering, Adv. Drug Deliv. Rev. 65 (2013) 1172-1187.

[14] R.S. Singh, N. Kaur, J.F. Kennedy, Pullulan and pullulan derivatives as promising biomolecules for drug and gene targeting, Carbohydr. Polym.123 (2015) 190-207.

[15] G.M. Whitesides, J.P. Mathias, C.T. Seto, Molecular self assembly and nanochemistry: a chemical strategy for the synthesis of nanostructures, Science 29 November 1991, 1312-1319.

[16] A.V. Kabanov, S.V. Vinogradov, Nanogels as pharmaceutical carriers: finite networks of infinite capabilities, Angew. Chem. Int. Ed. 48 (2009) 5418-5429.

[17] E. Montanari, S. Capece, C. Di Meo, M. Meringolo, T. Coviello, E. Agostinelli, P. Matricardi, Hyaluronic acid nanohydrogels as a useful tool for BSAO immobilization in the treatment of melanoma cancer cells, Macromol. Biosci. 13 (2013) 1185-1194.

[18] N. Zhang, P.R. Wardwell, R. A. Bader, Polysaccharide-based micelles for drug delivery, Pharmaceutics 5 (2013) 329-352.

[19] K. Akiyoshi, H. Takanabe, Te. Sato, To. Sato, H. Kondo, J. Sunamoto, Cell Specificity of Polysaccharide Derivatives on Liposomal Surface, J. Chem. Lett. 19 (1990) 473-476. 
[20] J. Sunamoto, T. Sato, T. Taguchi , H. Hamazaki, Naturally occurring polysaccharide derivatives which behave as an artificial cell wall on an artificial cell liposome, Macromolecules 25 (1992) 5665-5670.

[21] K. Akiyoshi, S. Yamaguchi, J. Sunamoto, Self-aggregates of hydrophobic polysaccharide derivatives, Chem. Lett. 20 (1991) 1263-1266.

[22] K. Akiyoshi, K. Nagai, T. Nishikawa, J. Sunamoto, Self-aggregates of hydrophobized polysaccharide as a host for macromolecular guests, Chem. Lett. 21 (1992) 1727-1730.

[23] K. Akiyoshi, S. Deguchi , N. Moriguchi, S. Yamaguchi, J. Sunamoto, Self-aggregates of hydrophobized polysaccharides in water. Formation and characteristics of nanoparticles, Macromolecules 26 (1993) 3062-3068.

[24] K. Kuroda, K. Fujimoto, J. Sunamoto, K. Akiyoshi, Hierarchical self-assembly of hydrophobically modified pullulan in water: gelation by networks of nanoparticles, Langmuir 18 (2002) 3780-3786.

[25] T. Nishikawa, K. Akiyoshi, J. Sunamoto, Supramolecular assembly between nanoparticles of hydrophobized polysaccharide and soluble protein complexation between the self-aggregate of cholesterol-bearing pullulan and $\alpha$-chymotrypsin, Macromolecules 27 (1994) 7654-7659.

[26] K. Akiyoshi, T. Nishikawa, S. Shichibe, J. Sunamoto, Stabilization of insulin upon supramolecular complexation with hydrophobized polysaccharide nanoparticle, Chem. Lett. 24 (1995) 707-708.

[27] K. Akiyoshi, S. Kobayashi, S. Shichibe, D. Mix, M. Baudys, S. W. Kim, J. Sunamoto, Selfassembled hydrogel nanoparticle of cholesterol-bearing pullulan as a carrier of protein drugs: complexation and stabilization of insulin, J. Contr. Rel. 54 (1998) 313-320.

[28] S-i. Sawada, K. Akiyoshi, K., Nano-encapsulation of lipase by self-assembled nanogels: induction of high enzyme activity and thermal stabilization, Macromol. Biosci. 10 (2010) 353-358.

[29] Y. Sasaki, K. Akiyoshi, Nanogel engineering for new nanobiomaterials: from chaperoning engineering to biomedical applications, Chem. Rec. 10 (2010) 366-376. 
[30] K. Akiyoshi, Y. Sasaki, J. Sunamoto, Molecular chaperone-like activity of hydrogel nanoparticles of hydrophobized pullulan: thermal stabilization with refolding of carbonic anhydrase B, Bioconj. Chem. 10 (1999) 321-324.

[31] Y. Nomura, M. Ikeda, N. Yamaguchi, Y. Aoyama, K. Akiyoshi, Protein refolding assisted by self-assembled nanogels as novel artificial molecular chaperone, FEBS Letters 553 (2003) 271-276.

[32] S-i. Sawada, Y. Nomura, Y. Aoyama, K. Akiyoshi, Heat shock protein-like activity of nanogel artificial chaperone for citrate synthase, J. Bioact. Compat. Polym. 21 (2006) 487-501.

[33] S-i. Sawada, Y. Sasaki, Y. Nomura, K. Akiyoshi, Cyclodextrin-responsive nanogel as an artificial chaperone for horseradish peroxidase, Colloid Polym.Sci. 289 (2011) 685-691.

[34] K. Ikeda, T. Okada, S-i. Sawada, K. Akiyoshi, K. Matsuzaki, Inhibition of the formation of amyloid $\beta$-protein fibrils using biocompatible nanogels as artificial chaperone, FEBS Letters, 580 (2006) 6587-6595.

[35] N. Inomoto, N. Osaka, T. Suzuki, U. Hasegawa, Y. Ozawa, H. Endo, K. Akiyoshi, M. Shibayama, Interaction of nanogel with cyclodextrin or protein: Study by dynamic light scattering and small-angle neutron scattering, Polymer 50 (2009) 541-546.

[36] M. Gao, S. Toita, S-i. Sawada, K, Akiyoshi, B. Torger Stokke, Cyclodextrin triggered dimensional changes of polysaccharide nanogel integrated hydrogels at nanometer resolution, Soft Matter 9 (2013) 5178-5185.

[37] M. Yokota, Y. Kobayashi, J. Morita, H. Suzuki, Y. Hashimoto, Y. Sasaki, K. Akiyoshi, K. Moriyama, Therapeutic effect of nanogel-based delivery of soluble FGFR2 with S252W mutation on craniosynostosis, PLOS ONE, published 08 Jul 2014, DOI: 10.1371/journal.pone.0101693.

[38] Y-II. Jeong, J-W. Nah, H-K. Na, K. Na, I-S. Kim, C-S. Cho, S-H Kim, Self assembling nanospheres of hydrophobized pullulans in water, Drug. Dev. Ind. Pharm. 25 (1999) 917-927. 
[39] S-W. Jung, Y-II. Jeong,Y-H. Kim, S-H. Kim, Self-assembled polymeric nanoparticles of poly(ethylene glycol) grafted pullulan acetate as a novel drug carrier, Arch. Pharm. Res. 27 (2004) $562-569$.

[40] W. Henni-Silhadi, M. Deyme, M.M. Boissonnade, M. Appel, D. Le Cerf, L. Picton, V. Rosilio, Enhancement of the solubility and efficacy of poorly water-soluble drugs by hydrophobicallymodified polysaccharide derivatives, Pharm. Res. 24, (2007) 2317-2325.

[41] M. Dionisio, C. Cordeiro, C. Remunan-Lopez, B. Seijo, A.M. Rosa da Costa, A. Grenha, Pullulan-based nanoparticles as carriers for transmucosal protein delivery, Eur. J. Pharm. Sci. 50 (2013) 102-113.

[42] H. Ringsdorf , J. Venzmer , F.M. Winnik, Fluorescence studies of hydrophobically modified poly(N-isopropylacrylamides), Macromolecules 24 (1991) 1678-1686.

[43] K. Akiyoshi, E-C. Kang, S. Kurumada, J. Sunamoto, T. Principi, F.M. Winnik, Controlled association of amphiphilic polymers in water: thermosensitive nanoparticles formed by selfassembly of hydrophobically modified pullulans and poly( $N$-isopropylacrylamides), Macromolecules 33, (2000) 3244-3249.

[44] N. Morimoto, F.M. Winnik, K. Akiyoshi, Botryoidal assembly of cholesteryl-pullulan/ poly(Nisopropylacrylamide) nanogels, Langmuir 23 (2007) 217-223.

[45] N. Morimoto, T. Ohki, K. Kurita, K. Akiyoshi, Thermo-responsive hydrogels with nanodomains: rapid shrinking of a nanogel-crosslinking hydrogel of poly( $\mathrm{N}$-isopropyl acrylamide), Macromol. Rapid. Comm. 29 (2008) 672-676.

[46] N. Morimoto, S. Hirano, H. Takahashi, S. Loethen, D.H. Thompson, K. Akiyoshi, Selfassembled $\mathrm{pH}$-sensitive cholesteryl pullulan nanogel as a protein delivery vehicle, Biomacromolecules 14 (2013) 56-63.

[47] J-K. Cho, W. Park, K. Na, Self-organized nanogels from pullulan-g-poly(L-lactide) synthesized by one-pot method: physicochemical characterization and in vitro doxorubicin release, J. Appl. Polym. Sci. 113 (2009) 2209-2216. 
[48] S. Seo, C-S. Lee, Y-S. Jung, K. Na, Thermo-sensitivity and triggered drug release of polysaccharide nanogels derived from pullulan-g-poly(L-lactide) copolymers, Carbohydr. Polym. 87 (2012) 1105-1111.

[49] Y. Wang, Ya. Liu, Yu. Liu, Y. Wang, J. Wu, R. Li, J. Yang, N. Zhang, pH-sensitive pullulanbased nanoparticles for intracellular drug delivery, Polym. Chem. 5 (2014) 423-432.

[50] K. Na, Y. H. Bae, Self-assembled hydrogel nanoparticles responsive to tumor extracellular $\mathrm{pH}$ from pullulan derivative/sulfonamide conjugate: characterization, aggregation, and adriamycin release in Vitro, Pharm. Res. 19 (2002) 681-688.

[51] K-H. Park, H-C. Song, K. Na, H-S. Bom, K.H. Lee, S. Kim, D. Kang, D. H. Lee, Ionic strength-sensitive pullulan acetate nanoparticles (PAN) for intratumoral administration of radioisotope: Ionic strength-dependent aggregation behavior and ${ }^{99 \mathrm{~m}}$ Technetium retention property, Coll. Surf. B: Biointerfaces 59 (2007) 16-23.

[52]. S. J. Lee,G-Y. Hong, Y-I. Jeong, M-S. Kang, J-S. Oh, C-E. Song, H.C. Lee, Paclitaxelincorporated nanoparticles of hydrophobized polysaccharide and their antitumor activity, Int. J. Pharm. 433 (2012) 121-128.

[53] J. Wang, S. Cui, Y. Bao, J. Xing, W. Hao, Tocopheryl pullulan-based self assembling nanomicelles for anti-cancer drug delivery, Mater. Sci. Eng. C, 43 (2014) 614-621.

[54] H. Li, Y. Cui, J. Liu, S. Bian, J. Liang, Y. Fan, X. Zhang, Reduction breakable cholesteryl pullulan nanoparticles for targeted hepatocellular carcinoma chemotherapy, J. Mater. Chem. B, 2 (2014) 3500-3510.

[55] Yi. Wang, H. Chen, Y. Liu, J. Wu, P. Zhou, Ya. Wang, R. Li, X. Yang, N. Zhang, $\mathrm{pH}$-sensitive pullulan-based nanoparticle carrier of methotrexate and combretastatin $\mathrm{A} 4$ for the combination therapy against hepatocellular carcinoma, Biomaterials 34 (2013) 7181-7190. 
[56]. H. Guo, Y. Liu, Y. Wang, J. Wu, X. Yang, R. Li, Y. Wang, N. Zhang, pH-sensitive pullulanbased nanoparticle carrier for adriamycin to overcome drug-resistance of cancer cells, Carbohydr. Polym.(2014) 908-917.

[57] Y. Liu, Ya. Wang, C. Zhang, P. Zhou, Y. Liu, T. An, D. Sun, N. Zhang, Yi. Wang, Core-Shell nanoparticles based on pullulan and poly( $\beta$-amino) ester for hepatoma-targeted codelivery of gene and chemotherapy agent, ACS Appl. Mater. Interfaces, 6 (2014) 18712-18720.

[58] H. Li, S. Bian, Y. Huang, J. Liang, Y. Fan, X. Zhang, High drug loading pH-sensitive pullulanDOX conjugate nanoparticles for hepatic targeting, J. Biomed. Mater. Res. Part A, 102 (2014) 150159.

[59] S. Kim, K.M. Park, J.Y. Ko, I.C. Kwon, H.G. Cho, D. Kang, I.T.Yu, K. Kim , K. Na, Minimalism in fabrication of self-organized nanogels holding both anti-cancer drug and targeting moiety, Colloids Surfaces B, 63 (2008) 55-63.

[60] H. Zhang, Fei Li, J. Yi, C. Gu, L.Fan, Y. Qiao, Y. Tao, C. Cheng, H. Wu, Folate-decorated maleilated pullulan-doxorubicin conjugate for active tumor-targeted drug delivery, Eur. J. Pharm. Sci. 42 (2011) 517-526.

[61] F. Li, H. Zhang, C Gu, Li Fan, Y. Qiao, Y. Tao, Chong Cheng, Hong Wu, Jun Yi, Selfassembled nanoparticles from folate-decorated maleilated pullulan-doxorubicin conjugate for improved drug delivery to cancer cells, Polym. Int. 62 (2013) 165-171.

[62]B-c. Bae, K. Na, Self-quenching polysaccharide-based nanogels of pullulan/folatephotosensitizer conjugates for photodynamic therapy, Biomaterials 31 (2010) 6325- 6335.

[63] W. Yang, M. Wang, L. Ma, H. Li, L. Huang, Synthesis and characterization of biotin modified cholesteryl pullulan as a novel anticancer drug carrier, Carbohydr. Polym. 99 (2014) 720-727.

[64] H. Ayame, N. Morimoto, K. Akiyoshi, Self-assembled cationic nanogels for intracellular protein delivery, Bioconj. Chem. 19 (2008) 882-890.

[65] K. Watanabe, Y. Tsuchiya, Y. Kawaguchi, S-i. Sawada, H. Ayame, K. Akiyoshi, T. Tubata, 
The use of cationic nanogels to deliver proteins to myeloma cells and primary $\mathrm{T}$ lymphocytes that poorly express heparan sulphate, Biomaterials 32 (2011) 5900-5905.

[66] U. Hasegawa, S-i. M. Nomura, S.C. Kaul, T. Hirano, K. Akiyoshi, Nanogel-quantum dot hybrid nanoparticles for live cell imaging, Biochem. Bioph. Res. Co. 331 (2005) 917-921.

[67] S. Maya, B. Sarmento, A. Nair, N.S. Rejinold, S.V. Nair, R. Jayakumar, Smart stimulisensitive nanogels in cancer drug delivery and imaging: a review, Curr. Pharm. Design, 19 (2013) 7203-7218.

[68] L. Cheng, C. Wang, X. Ma, Q. Wang, Y. Cheng, H. Wang, Y. Li, Z. Liu, Multifunctional upconversion nanoparticles for dual-modal imaging-guided stem cell therapy under remote magnetic control, Adv. Func. Mater. 23 (2013) 272-280.

[69]. K.S. Kim, W. Park, K. Na, Gadolinium-chelate nanoparticle entrapped human mesenchymal stem cell via photochemical internalization for cancer diagnosis, Biomaterials 36 (2015) 90-97.

[70] S.A. Ferreira, F.M. Gama, M. Vilanova, Polymeric nanogels as vaccine delivery systems, Nanomedicine: NBM, 9 (2013) 159-173.

[71] S. Kitano, S. Kageyama, Y. Miyahara, A. Hiasa, H. Naota, S. Okumura, H. Imai, T. Shiraishi, M. Masuya, M. Nishikawa, J. Sunamoto, K. Akiyoshi, T. Kanematsu, A.M. Scott, R. Murphy, E.W. Hoffman, L.J. Old, H. Shiku, HER2-specific T-cell immune responses in patients vaccinated with truncated HER2 protein complexed with nanogels of cholesteryl pullulan, Clin. Cancer Res. 12 (2006) 7397-7405.

[72] S. Kageyama, S. Kitano, M. Hirayama,Y. Nagata, H. Imai,T. Shiraishi, K. Akiyoshi, A.M. Scott, R. Murphy, E.W. Hoffman, L.J. Old, N. Katayama, H. Shiku, Humoral immune responses in patients vaccinated with 1-146 HER2 protein complexed with cholesteryl pullulan nanogel, Cancer Sci. 99 (2008) 601-607.

[73] H. Shiku, L. Wang, Y. Ikuta, T. Okugawa, M. Schmitt, X. Gu, K. Akiyoshi, J. Sunamoto, H. Nakamura, Development of a cancer vaccine: peptides, proteins, and DNA, Cancer Chemother. Pharm. 46 Suppl. (2000) S77-S82. 
[74] K. Hasegawa, Y. Noguchi, F. Koizumi, A. Uenaka, M. Tanaka, M. Shimono, H. Nakamura, H. Shiku, S. Gnjatic, R. Murphy, Y. Hiramatsu, L.J. Old, E. Nakayama, In vitro Stimulation of CD8 and CD4 T cells by dendritic cells loaded with a complex of cholesterol-bearing hydrophobized pullulan and NY-ESO-1 protein: identification of a new HLA-DR15-binding CD4 T-cell epitope Clin. Cancer Res. 12 (2006) 1921-1927.

[75] A. Uenaka, H. Wada, M. Isobe, T. Saika, K. Tsuji, E. Sato, S. Sato, Y. Noguchi, R. Kawabata, T. Yasuda, Y. Doki, H. Kumon, K. Iwatsuki, H. Shiku, M. Monden, A.A. Jungbluth, G. Ritter, R. Murphy, E. Hoffman, L.J. Old, E. Nakayama, T cell immunomonitoring and tumor responses in patients immunized with a complex of cholesterol-bearing hydrophobized pullulan (CHP) and NYESO-1 protein, Cancer Immunity 7 : 9 (19 April 2007).

[76] M. Aoki, S. Ueda, H. Nishikawa, S. Kitano, M. Hirayama, H. Ikeda, H. Toyoda, K. Tanaka, M. Kanai, A. Takabayashi, H. Imai, T. Shiraishi, E. Sato, H. Wada, E. Nakayama, Y. Takei, N. Katayama, H. Shiku, S. Kageyama, Antibody responses against NY-ESO-1 and HER2 antigens in patients vaccinated with combinations of cholesteryl pullulan (CHP)-NY-ESO-1 and CHP-HER2 with OK-432, Vaccine 27 (2009) 6854-6861.

[77]. S. Kageyama, H. Wada, K. Muro, Y. Niwa, S. Ueda, H. Miyata, S. Takiguchi, S.H. Sugino, Y. Miyahara, H. Ikeda, N. Imai, E. Sato, T. Yamada, M. Osako, M. Ohnishi, N. Harada, T. Hishida, Y. Doki, H. Shiku, Dose-dependent effects of NY-ESO-1 protein vaccine complexed with cholesteryl pullulan (CHP-NY-ESO-1) on immune responses and survival benefits of esophageal cancer patients, J. Trans. Med., 11 (2013) 246 (5 October 2013).

[78] T. Shimizu, T. Kishida, U. Hasegawa, Y. Ueda, J. Imanishi, H. Yamagishi, K. Akiyoshi, E. Otsuji, O. Mazda, Nanogel DDS enables sustained release of IL-12 for tumor immunotherapy, Biochem. Bioph. Res. Co. 367 (2008) 330-335.

[79] I. Gyu Kong, A. Sato, Y. Yuki, T. Nochi, H. Takahashi, S-i. Sawada, M. Mejima, S. Kurokawa, K. Okada, S. Sato, D.E. Briles, J. Kunisawa, Y. Inoue, M. Yamamoto, K. Akiyoshi, H. Kiyono, Nanogel-Based PspA Intranasal Vaccine Prevents Invasive Disease and Nasal Colonization by Streptococcus pneumoniae, Infect. Immun. 81 (2013) 1625-1634. 
[80] T. Nochi, Y. Yuki, H. Takahashi, S-i. Sawada, M. Mejima, T. Kohda, N. Harada, I. Gyu Kong, A. Sato, N. Kataoka, D. Tokuhara, S. Kurokawa, Y. Takahashi, H. Tsukada, S. Kozaki, K. Akiyoshi, H. Kiyono, Nanogel antigenic protein-delivery system for adjuvant-free intranasal vaccines, Nature Mater. 9 (2010) 572-578.

[81] Y. Yukia, T. Nochi, I.Gyu Kong, H. Takahashi, S-i. Sawada, K. Akiyoshi, H. Kiyono, Nanogel-based antigen-delivery system for nasal vaccines, Biotec. Gen. Eng. Rev. 29 (2013) 61-72.

[82] D. Muraoka, N. Harada, T. Hayashi, Y. Tahara, F. Momose, S-i. Sawada, S-a. Mukai, K. Akiyoshi, H. Shiku, Nanogel-based immunologically stealth vaccine targets macrophages in the medulla of lymph node and induces potent antitumor immunity, ACS Nano, 8 (2014) 9209-9218.

[83] Y-S. Jung, W. Park, K. Na, Temperature-modulated noncovalent interaction controllable complex for the long-term delivery of etanercept to treat rheumatoid arthritis, J. Contr. Rel. 171 (2013) 143-151.

[84] J.-H. Kang, Y. Tachibana, W. Kamata, A. Mahara, M. Harada-Shiba, T. Yamaoka, Livertargeted siRNA delivery by polyethylenimine (PEI)-pullulan carrier, Bioorg. Med. Chem., 18 (2010) 3946-3950.

[85] M.R. Rekha, Chandra P. Sharma, Hemocompatible pullulan-polyethyleneimine conjugates for liver cell gene delivery: In vitro evaluation of cellular uptake, intracellular trafficking and transfection efficiency, Acta Biomat. 7 (2011) 370-379.

[86] C. Hayashi, U. Hasgawa,Y. Saita, H. Hemmi, T. Hayata, K. Nakashima, Y. Ezura, T. Amagasa, K. Akiyoshi, M. Noda Osteoblastic bone formation is induced by using nanogel-crosslinking hydrogel as novel scaffold for bone growth factor, J. Cell. Physiol., 220 (2009) 1-7.

[87] M.Fujioka-Kobayashi, M.S. Ota, A. Shimoda, K-i. Nakahama, K. Akiyoshi, Y. Miyamoto, S. Iseki, Cholesteryl group- and acryloyl group-bearing pullulan nanogel to deliver BMP2 and FGF18 for bone tissue engineering, Biomaterials, 33 (2012) 7613-7620.

[88] A. Shimoda, Y. Yamamoto, S-i. Sawada, K. Akiyoshi, Biodegradable nanogel-integrated hydrogels for sustained protein delivery, Macromol. Res. 20 (2012) 266-270. 
[89] Y. Hashimoto, S-a. Mukai, S-i. Sawada, Y. Sasaki, K. Akiyoshi, Nanogel tectonic porous gel loading biologics, nanocarriers, and cells for advanced scaffold, Biomaterials, 37 (2015) 107-115. 